\title{
Criminologie
}

\section{Dialogue avec André Normandeau}

\section{Georges Paradis}

Volume 10, numéro 2, 1977

La criminologie au Québec

URI : https://id.erudit.org/iderudit/017076ar

DOI : https://doi.org/10.7202/017076ar

Aller au sommaire du numéro

Éditeur(s)

Les Presses de l'Université de Montréal

ISSN

0316-0041 (imprimé)

1492-1367 (numérique)

Découvrir la revue

Citer ce document

Paradis, G. (1977). Dialogue avec André Normandeau. Criminologie, 10(2),

78-92. https://doi.org/10.7202/017076ar d'utilisation que vous pouvez consulter en ligne.

https://apropos.erudit.org/fr/usagers/politique-dutilisation/ 


\section{DIALOGUE AVEC \\ ANDRÉ NORMANDEAU}

Propos recueillis par : Georges Paradis

Pourriez-vous nous livrer quelques éléments de votre vie personnelle et quelques motifs justifiant, selon vous, votre option pour la criminologie?

Je suis né en 1942 en banlieue de Montréal, Verdun, Québec. Alors que j'avais un an, mes parents déménagèrent à Ste-Rose qui est maintenant un quartier de Ville de Laval et qui, à ce moment-là, était une petite ville de campagne. À l'âge de six ans, nous avons déménagé de nouveau à Verdun, et là, $\mathrm{j}$ 'ai fait mon école élémentaire. Pour mon secondaire et collégial (1954-1962) je me suis retrouvé pensionnaire au collège classique de l'Assomption. Entre-temps, mes parents déménagèrent à Rosemont, un quartier de Montréal. À 20 ans, immédiatement après mon classique, je suis entré en sociologie à l'Université de Montréal (1962-1964). Dans la sociologie, je cherchais un domaine appliqué, par exemple: la sociologie familiale, la sociologie du travail, la criminologie... Finalement pour des raisons de hasard, de lecture et d'intérêt personnel, j'ai choisi la criminologie. J'ai reçu une bourse, la Woodrow Wilson (d'origine américaine) me permettant de poursuivre des études en sociologie, ou dans un domaine connexe, à la condition d'aller dans une université américaine. Je suis donc passé directement du baccalauréat en sociologie de l'Université de Montréal à la Maîtrise en criminologie à l'Université de la Pennsylvanie, Philadelphie (1964-1965) et au doctorat en sociologie criminelle a la même université (1965-1968).

Qu'est-ce que vous retirez de votre formation classique?

Nous * jouions * beaucoup à ce moment-là, à l'Assomption comme d'ailleurs dans la plupart des collèges classiques, «à l'intellectuel ». Nous voulions absolument tout lire. En * Belles-Lettres, nous lisions à peu près tous les écrits des grands romanciers des XVIre, XIXe et $\mathbf{X X}^{e}$ siècles. Nous étions avides de maîtriser les idées d'un auteur et d'en discuter avec d'autres. Le fait que quatre-vingt-dix pour cent des étudiants du collège de l'Assomption étaient pensionnaires favorisait cet échange intellectuel puisque nous vivions constamment ensemble. $\mathrm{Si}$ je compare le collège classique au CEGEP d'aujourd'hui, je pense, sans l'affirmer de façon péjorative, que le classique créait un climat intellectuel pluss profond.

Quels sont les écrivains qui ont attiré le plus votre attention?

Au niveau de la littérature, au sens classique du terme, ce sont les écrivains dits « catholiques > que j'ai surtout appréciés. Parmi les grands écrivains, je retiens Graham Greene, Bernanos et Julien Green. Ces auteurs décrivent sous de multiples facettes une série de problèmes moraux et religieux qui m'ont toujours intéressé.

A ce moment-là, opter pour la sociologie n'était-ce pas précisément opter pour laïciser cette perspective religieuse?

Je ne le pense pas. La sociologie de Montréal au début des années 60 , au moment où je m'y suis intéressé, était encore très imprégnée du modèle des sciences sociales d'inspiration «morale "développé dans les 
années 40 et 50 à l'Université Laval (Québec) par le P. Lévesque. Le département de sociologie de l'Université de Montréal venait à peine, d'ailleurs, d'être organisé (1958). La plupart des professeurs de sociologie à Montréal avaient été mêlés aux organisations religieuses ou parareligieuses des années 40 et 50 . Les sciences sociales, y inclus la sociologie, étaient en fait, au début, une extension laïque des idées sociales et économiques développées dans les encycliques de l'Église catholique. Les sciences sociales, de façon générale, étaient souvent rattachées à l'idée de * vocation ", au même sens que la prêtrise. C'est ainsi que mes premières préoccupations personnelles en sociologie s'inscrivaient dans cette perspective. Dès mon premier semestre en sociologie, mon travail principal porta sur la secte de St-Jovite, constituée de * catholiques excommuniés s.

Pourtant en 1963, vous acceptiez de participer activement à la revue Cité libre, dirigée par Pierre-Elliott Trudeau. Comment perceviez-vous cette revue ?

Au moment où j'ai fait mon classique, Cité libre passait pour une revue d'extrême gauche. Le Premier Ministre du Québec dans les années 50, Maurice Duplessis avait même lancé des accusations de * communisme, contre Trudeau et ses collaborateurs. C'est donc une revue que nous avons lue en cachette jusqu'au début des années 60 . Il faut dire que de 60 à 65 , Cité libre se retrouve un peu en porte à faux parce que la grande bataille qu'ils ont menée dans les années 50 est terminée avec la mort de Duplessis (1959) et la défaite électorale de son parti (1960). Toutefois, Cité libre demeure alors une revue sociale-démocrate de combat social. Le combat m'intéressait vivement. Dire qu'à cette époque nous étions conscients d'une * révolution * serait exagéré. Nous vivions cette période comme une évolution aux cadences accélérées.

Pour vous, quels sont ceux qui représentaient la gauche à ce moment-là ?

$\mathrm{Au}$ point de vue politique, c'était le Nouveau-Parti-Démocratique (N.P.D.). A Cité libre, par exemple, on appuyait ouvertement le N.P.D. Le N.P.D. était issu du C.C.F., un parti plus ouvertement socialiste. $\mathrm{Au}$ point de vue culturel, il n'y avait pas de mouvement spécifiquement dit de gauche. Le * culturel s se traduisait tout simplement par une très grande liberté d'expression. Tous les gens avaient l'impression de pouvoir enfin s'exprimer. Je ne me souviens pas avoir été e marqué s par un mouvement littéraire ou culturel spécifique. Tout m'apparaissait comme nouveau et intéressant. C'était un vaste mouvement de libération. Pour moi, c'était ça, la gauche. En fait, la gauche, c'est le contraire du c fascisme s. Le fascisme, pour moi, est un manque de connaissance; la gauche, c'est * nommer les choses *, comme le disait Moravia.

Croyez-vous que cette *évolution * brusque du contexte québécois a changé quelque chose aux sciences sociales et à la sociologie?

Oui, puisque, effectivement, la sociologie, du moins à Montréal, s'est réorientée profondément entre les années 1960 et 1965 . D'une sociologie cmorale - d'orientation appliquée, l'on est passé à une sociologie réflexive et critique. D'une sociologie pour réformateurs, l'on est passé à une sociologie radicale. Personnellement, je suis demeuré fidèle à la première orientation, celle d'une sociologie appliquée, de type réformatrice. C'est ainsi que j'ai travaillé au département de sociologie en 1963 et 1964 au sein de groupes de recherches appliquées sur la famille. 
Quels sont les thèmes ou les auteurs sociologiques qui vous interessaient d'une façon spéciale?

Je suis très "classique à ce sujet. Je me raccroche au modèle - structurel-fonctionnel s américain, style Talcott Parsons et Robert Merton. J'ai eu beaucoup de plaisir, en particulier, à lire les œuvres de Merton. Son étude du patronage politique m'avait beaucoup impressionné. Sa description du phénomène de la prédiction créatrice (s ce qui est défini comme réel est réel dans ses conséquences ») m'avait permis, également, de faire la jonction entre les explications psychologiques et sociologiques des phénomènes humains et sociaux. Une autre idée intéressante de Merton est celle de la esérendipidité, de cette compréhension fortuite d'un phénomène après une recherche théorique et empirique en profondeur. Il ne faut pas oublier, non plus, les tentatives de Merton pour développer des théories à moyenne portées. Les principales théories criminologiques se rattachent d'ailleurs à ce modèle. Le théoriciencriminologue qui m'a le plus marqué est Georges Vold, grâce à ses tentatives d'intégrer le modèle structurel-fonctionnel au modèle conflictuel et marxiste.

Toutefois, il faut admettre qu'en criminologie il n'y a pas de grands théoriciens. Les théories associées aux noms de Shaw et McKay, Sutherland, Sellin, Cohen, Cloward, Ohlin et Matza sont en fait des explications partielles de la délinquance et de la criminalité.

J'ai beaucoup apprécié, finalement, les écrits de C.W. Mills, particulièrement ses réflexions critiques au sujet de la nécessité de développer l'imagination sociologique. Un parallèle avec l'imagination et la créativité criminologique serait utile à tracer à partir des idées de Mills.

Dans un de vos articles sur \& l'homme psychologique et la culture du $X X X^{e}$ siècle $\gg$, vous affirmiez ce qui suit : < La civilisation occidentale a produit quatre principaux types d'homme : l'homme politique, économique, religieux et psychologique. Ceux-ci ont à tour de rôle, maís aussi de façon souvent concomitante, jalonné l'histoire. , Quel(s) type(s) d'homme croyezvous représenter ou mieux avec quel(s) type(s) d'homme avez-vous le plus d'aftinité ?

Je suis beaucoup plus à l'aise avec l'homme politique et l'homme économique. Pourtant, je devrais aussi être à l'aise avec l'homme religieux de par ma formation au collège classique. À titre individuel, d'ailleurs, je suis toujours demeuré un homme religieux. Toutefois, cet homme est - mon petit roi " caché, personnel, intériorisé. Je suis donc différent de l'homme religieux d'autrefois qui projetait à travers ses valeurs religieuses une façon d'interpréter l'homme politique et l'homme économique. Quant à l'homme psychologique, produit du vingtième siècle, je le regarde de l'extérieur car je n'ai pas atteint personnellement ce niveau où je serais tellement détaché du politique et de l'économique que je pourrais dire que j'ai une liberté intérieure totale, indépendante des valeurs politiques, économiques et religieuses.

Si vous aviez à tirer une idée directrice de votre production intellectuelle, que serait-elle?

Ca, c'est risqué. Quand on a 35 ans, il est prématuré, à mon avis, d'établir une telle idée-mâ̂tresse. Il s'agit d'un problème de maturité intellectuelle. Cette maturité s'exprime par un recul que je ne peux pas avoir car je suis encore * collé , à l'action. « Ma s production a été, en fait, un pot-pourri plutôt qu'une série d'étapes qui seraient bien délimitées. 
Toutefois, depuis deux ou trois ans, je m'intéresse davantage à la politique criminelle. J'essaie de trouver des modèles de réforme du système d'administration de la justice en matière pénale et criminelle en m'appuyant sur les théories criminologues et les résultats des recherches empiriques. Je n'ai pas encore trouvé une idée explicite en politique criminelle. Cependant, j'ai nettement un e parti-pris : idéologique pour une justice sociale plus égalitaire et il est probable que ce choix influence et influencera le sens et la portée de ma production intellectuelle au chapitre de la politique criminelle dans mes travaux des prochaines années. Mon objectif personnel est de contribuer à un élargissement sensible du corridor de la justice sociale. Mes études et mes recherches en politique criminelle en seront sûrement marquées.

$\hat{A}$ vingt ans, j'ai cru qu'une justice sociale plus égalitaire n'était possible que par le moyen d'une révolution. Par la suite, je ne sais si c'est un phénomène de vieillissement prématuré ou de maturité précoce, j'ai opté résolument pour un modèle réformateur où le changement social est axé sur une série de réformes successives. Je dis souvent aux étudiants que mon but est de changer 10 à 20 pour cent du système. En effet, je pense que c'est la seule façon d'élargir le corridor de la justice en respectant le processus démocratique. Cette option est très profonde chez moi. À titre personnel, je voudrais bien à l'occasion changer 100 pour cent du système. Toutefois, une éthique des moyens m'empêche de recourir à des moyens radicaux et révolutionnaires car je refuse de changer le système sans l'accord d'une majorité de la population. Ce que je suis prêt à faire, c'est un long travail d'information auprès de la population et de ses dirigeants afin de faire évoluer notre système de justice.

Est-ce que le crime est une réalité pour vous ?

Le crime est à la fois une réalité et une fiction. C'est une réalité lorsque tel ou tel acte est défendu par une majorité de gens qui refuse que le vol ou la violence deviennent un moyen datteindre un but ou un objectif personnel ou social. C'est une fiction lorsque la définition légale des actes criminels est élaborée par un groupe minoritaire qui a le pouvoir d'imposer ses volontés et ses visions du « bien et du mal ?. Par exemple, si les gens de milieux socio-économiques défavorisés sont condamnés à la prison pour vol alors que les gens de milieux favorisés reçoivent seulement des amendes pour fraudes, la loi devient alors une fiction. Cette fiction, toutefois, peut être analysée comme une réalité. Un dilemme important se pose ici. Si nous changions la loi et l'administration de la justice afin de sévir contre la criminalité dite à col blanc, nous proposerions en fait une criminalisation des classes sociales, moyennes et favorisées. Cela me pose un problème parce que, si par ailleurs, les criminologues essaient de décriminaliser en partie la criminalité classique des classes sociales défavorisées, ne sommes-nous pas en train de renverser simplement la pyramide ou la pointe de l'iceberg et de remplacer une intolérance par une autre ? Mon objectif personnel est d'élargir le corridor de tolérance à l'ensemble des citoyens car la justice plus égalitaire aura de meilleures chances de se développer dans un tel contexte. Aussi, faut-il trouver un moyen de concilier la réalité du crime avec sa fiction.

Le crime, * réalité * et * fiction *, peut-il, toutefois, être un objet d'étude ? Émile Durkheim disait que la sociologie était l'étude des faits sociaux envisagés comme des < choses *. Or, le crime, réalité et fiction, est un fait social par excellence, et la science criminologique est précisément 
axée sur l'étude des * choses * criminelles. J'ai écrit en 1967, un texte ( $\&$ La Criminologie : pot-pourri ou discipline scientifique distincte ? $>$ ) ou je définissais la criminologie comme science, comme discipline. Je persiste à croire que la criminologie est fondamentalement une science descriptive et critique des phénomènes criminels, autant lorsque l'objet d'étude est le passage à l'acte (* criminologie clinique ») que celui de la réaction sociale (ะ politique criminelle $>$ ).

Toutefois, alors qu'il y a 10 ans, je ne croyais pas qu'un praticien de la criminologie était un véritable criminologue, je pense, aujourd'hui, que si la formation d'un criminologue praticien en matière scientifique est sérieuse, ce qui est le cas, à mon avis, ici à notre Ecole, cette réticence qui était mienne n'a plus de raison d'être.

$\mathrm{Si}$ le criminologue fait une bonne analyse des hommes et des institutions, une critique valable s'y rattachera. Et si la critique est solide, des pistes pour la "réforme du criminel et/ou du système de justice y seront greffées. Le criminologue doit travailler anx trois niveaux.

Lorsque vous regardez la production criminologique (recherches, études, mémoires...) de l'Ecole de criminologie de Montréal et que vous essayez de l'apprécier à travers le triple but que vous venez de souligner (analyse, critique et réforme), quelle impression en dégagez-vous?

Nous avons tout d'abord fait un bon travail au niveau de l'analyse quantitative des phénomènes criminels. Je prends, par exemple, le secteur de la délinquance juvénile, des travaux de recherches de Szabo et alii dans les années 60 jusqu'aux travaux des années 70 du Groupe de recherche en inadaptation juvénile de Cusson, Fréchette et LeBlanc. En revanche, l'analyse qualitative des phénomènes criminels est demeurée relativement marginale jusqu'à ces derniers temps. Heureusement, les travaux récents de Tardif et alii augurent bien pour l'avenir. Par contre, je ne voudrais pas que nous passions tout simplement d'un extrême à l'autre. Je pense qu'une bonne analyse, utile pour comprendre toutes les dimensions d'un phénomène, doit être à la fois qualitative et quantitative. Au niveau de la critique, je pense que nous avons été très faibles. Nous avons pris pour acquis que la criminologie, par sa seule existence, était à la fois analyse et critique des phénomènes. Or, ce n'est pas le cas. Une critique valable doit être structurée à partir d'une analyse sérieuse non seulement des faits criminels eux-mêmes, mais également des conditions sociales et économiques du milieu où les phénomènes se produisent. Nous avons hésité jusqu'ici, malheureusement, à élargir notre critique au-delà des paramètres traditionnels de la criminologie. Finalement, au niveau de la réforme, les travaux d'orientation "politique criminelle, ont souvent mené à des prises de position publiques utiles pour accélérer les changements du système de justice; de plus, si les travaux d'orientation clinique n'ont pas encore débouché suffisamment, à mon avis, sur des propositions de - réformes cliniques systématiques, les recherches actuelles de Fréchette, en particulier, pourraient contribuer à remettre en question les instruments cliniques traditionnels utilisés dans le champ de la délinquance juvénile sur les plans du diagnostic, du pronostic et du traitement.

Croyez-vous que la criminologie dite radicale remet en question votre vision du crime?

La criminologie radicale remplace, à toutes fins pratiques, l'etude classique de la criminalité, qui est définie via le code pénal, par une étude beaucoup plus vaste, de nature sociopolitique, qui met l'accent sur l'impé- 
rialisme, le capitalisme, le sexisme et le racisme. Comme citoyen, ces phénomènes m'intéressent et peuvent même m'amener à prendre position activement pour telle ou telie réforme, pour le développement d'un socialisme à visage humain, par exemple. Toutefois, si les paramètres de la criminologie devaient dorénavant se limiter à ces * nouveaux * champs d'investigations, j'avoue que je me poserais la question suivante: cette criminologie radicale n'est-elle pas, en fait, une spécialité de la science politique ou de la sociologie plutôt que de la criminologie? Mais si j'utilise l'expression * criminologie nouvelle " pour exprimer cette préoccupation renouvelée de certains criminologues pour une analyse systématique et critique de la criminalité des classes sociales moyennes et favorisées, tels le crime à col blanc, les fraudes économiques de tout acabit, la corruption politique..., alors, là, je suis pleinement d'accord. Dans ce sens, la vision du crime des criminologues de demain sera sensiblement modifiée.

Si vous deviez nous tracer les grands traits du criminologue qui correspond à vos idées, quel portrait en feriez-vous ? Serait-il à la fois homme de la * pratique * et homme de « science :?

Il est impossible, à mon avis, d'être à la fois un praticien et un scientifique qualifiés. En effet, l'un comme l'autre exigent pour atteindre un degré de qualité réelle une formation supérieure spécialisée et une expérience de terrain relativement prolongée. Or, il est impossible, sauf exception, de poursuivre en profondeur au niveau de la formation universitaire deux champs de spécialisation aussi différents; il en est de même au niveau de l'expérience pratique subséquente. Aussi, sans porter de jugement de valeur sur l'un ou l'autre type de criminologue, j'ai appris à respecter à leur pleine valeur autant le criminologue-praticien que le criminologuechercheur compétents. La compétence professionnelle est et doit être la seule marque de commerce du criminologue, quel que soit son terrain d'expertise.

Est-ce qu'il y a un aspect spécifique de la criminologie que vous avez développé sous votre mandat en tant que directeur de l'Ecole de criminologie de l'Université de Montréal, et qui serait, en quelque sorte, l'originalité de votre mandat par rapport à celui de votre prédécesseur, Denis Szabo?

L'originalité, s'il y en a une, est en fait une « continuité dans le changement ${ }^{*}$. Il n'y a pas véritablement de brisure entre les deux mandats, mais plutôt une consolidation et une précision des orientations de la décennie 60 . C'est ainsi qu'après bien des hésitations et des discussions souvent laborieuses, nous en sommes venus à reconnaître de facto deux concentrations criminologiques à l'École : a) la criminologie clinique et b) la politique criminelle. Il s'agit donc d'une criminologie du passage à l'acte, dans le premier cas, et d'une criminologie de la réaction sociale, dans le second cas.

La criminologie clinique a ses sources dans le domaine de la psychiatrie et de la psychologie criminelle, alors que la politique criminelle s'appuie sur la sociologie criminelle, la science politique et le droit criminel. Dans cette perspective, nous avons mis beaucoup d'effort et d'énergie à appuyer le secteur clinique par la mise au point d'une véritable structure de stages pratiques alors que le secteur de politique criminelle s'est surtout distingué par le développement de recherches fondamentales et appliquées ainsi que la naissance de deux centres de recherche, le Centre international de criminologie comparée (1969) et le Groupe de recherche en inadaptation juvénile (1973). 
La plupart des criminologues des pays étrangers qui connaissent notre structure « à deux têtes 》 ne comprennent pas comment les deux concentrations peuvent coexister au sein d'une même Ecole. En effet, à l'étranger, la plupart des unités d'enseignement criminologique sont orientées plutôt vers l'un ou l'autre secteur. Montréal se distingue à ce sujet en relevant le défi d'une coexistence pacifique entre les deux orientations fondamentales de la criminologie moderne. Ce n'est pas toujours facile, mais il en vaut la peine car je suis profondément convaincu que l'analyse, la critique et les réformes proposées par les criminologues doivent être appuyées dans la mesure de nos moyens sur cette double vision de la réalité criminelle.

Est-ce que cette double orientation de l'Ecole a modifié le corps professoral ?

C'est un peu le dilemme de la $<$ poule $>$ et de $<$ l'ouf $»$, Toutefois, il est certain que les engagements des professeurs ces dernières années ont été faits en partie avec le désir de maintenir l'équilibre entre les deux secteurs. En fait, il y a actuellement dix professeurs à temps plein dont l'intérêt principal se situe au niveau de l'orientation clinique et dix autres qui ont surtout un intérêt en politique criminelle. De plus, une dizaine de praticiens sont chargés à temps partiel de leçons ou de cours de nature clinique. J'ajouterais que chaque secteur n'est pas monolithique et qu'il est possible de retrouver une gamme d'orientations secondaires. Ainsi, au niveau de la politique criminelle, un professeur comme Marie-Andrée Bertrand travaille sur la base đ'une orientation idéologique radicale et néo-marxiste alors que, personnellement, je travaille avec un éclairage structuraliste et fonctionnel classique.

Vous affirmiez, en 1972, ce qui suit: A la limite, et je le dis sans y rattacher d'étiquette péjorative, une criminologie strictement critique nous mène, si nous sommes sincères, à la guérilla contre la justice criminelle. Si nous étions honnêtes, nous devrions prendre le maquis afin de faire éclater le système?. Premièrement, maintiendriez-vous encore cette affirmation? Deuxièmement, si nous transposions cette affirmation dans la perspective de l'École, est-ce que vous croyez qu'une criminologie critique soulèverait une sorte de guérilla intra-muros ?

Je ferais aujourd'hui la distinction suivante. Si un criminologue exerce son métier comme intellectuel et comme professeur d'université, il lui est loisible d'être extrêmement critique des institutions de justice sans nécessairement s'engager lui-même dans l'action. Toutefois, si une critique radicale dépasse le stage de l'analyse et l'amène à conclure que seule la - révolution •, au sens propre du terme, peut résoudre la crise de la justice, je pense qu'il a un devoir moral de s'engager dans une forme d'action qui, d'une façon ou d'une autre, sera équivalente à une guérilla non seulement judiciaire mais de nature politico-socio-économique. Mais si le criminologue est d'allégeance réformiste, cette action sera orientée vers des formes d'action modérée et sectorielle de nature évolutive tant sur le plan proprement criminologique qu'aux niveaux politique, social et économique.

Transposée au sein de l'École de criminologie, je pense que la distinction est également valable. Des criminologues radicaux et fonctionnalistes peuvent travailler ensemble dans la même unité de travail et permettre ainsi aux étudiants d'être confrontés à un pluralisme théorique et méthodologique fort sain tant que leurs analyses n'aboutissent pas à 
proposer des moyens révolutionnaires pour changer le système. Car, si tel était le cas, le criminologue radical devrait honnêtement prendre le maquis au sein de sa propre Ecole de criminologie puisque cette dernière fait, en partie, le jeu du système, en tentant de l'anéliorer plutôt que de le changer radicalement. Je soupçonne qu'une partie de l'explication de la disparition de l'école de criminologie de l'Université de la Californie à Berkeley est liée à cette dialectique.

Pour ma part, je ne pourrais pas travailler avec un criminologue radical qui aurait répudié l'analyse scientifique pour se faire le défenseur de la révolution. Je respecterais alors son option, mais il n'y aurait plus de coexistence pacifique possible. La réforme et la révolution exigent des moyens très différents et irréconciliables. Or je le répète, je suis un réformiste de cour et d'esprit.

Vous avez déjà écrit que la formation criminologique devait être une formation complémentaire à la sociologie, et à la psychologie. Maintiendriez-vous encore cette idée?

Non, l'expérience de l'École de criminologie de Montréal m'a convaincu qu'il était possible de donner une formation psychosociale valable en intégrant des enseignements de plusieurs disciplines de base. Toutefois, il est également sain de maintenir un certain nombre d'entrées, au niveau de la maîtrise en particulier, d'étudiants qui ont fait des études préliminaires dans des disciplines connexes à la criminologie, telles que la sociologie, la psychologie, le service social, le droit..., afin de permettre un choc et une confrontation permanente de nature interdisciplinaire des visions et des idées sur la délinquance, la criminalité et la justice.

Pensez-vous que le programme de criminologie de l'Ecole est sous-développé par rapport aux données criminologiques proprement québécoises disponibles pour des fins d'enseignement de recherche ?

Je crois que ce sous-développement existait largement au cours de la décennie 60. C'était l'époque du développement de l'École. Très peu d'études et de recherches avaient alors été faites sur la criminalité et la justice au Québec. L'enseignement puisait abondamment aux sources criminologiques américaines et européennes. Toutefois, un changement important s'est accompli avec la décennie 70 . Les professeurs et les chercheurs québécois ont maintenant produit des matériaux criminologiques québécois en qualité et en quantité, sans compter le nombre plus élevé de mémoires et de thèses estudiantines dont les résultats sont profondément enracinés et collés au contexte québécois. Cet enracinement ne doit pas, cependant, nous fermer sur nous-mêmes. Aussi je juge utile et sain que le corps professoral soit composé d'une majorité de Québécois (15) mais également d'un certain nombre de néo-Québécois (5), sans oublier les professeurs étrangers qui sont invités chaque année.

Est-ce que vous constatez un changement de la population étudiante de l'Ecole depuis une dizaine d'années?

Il est certain que le $x$ type > d'étudiants qui s'inscrit à notre Ecole depuis 1970 est très différent de celui des années 60. D'abord, parce qu'il s'agit d'une autre génération d'étudiants et il est normal que leurs "visions du monde " aient évolué comme celle de tous les citoyens québécois. De façon particulière, toutefois, il faut se rappeler que la majorité des étudiants que nous accueillons depuis quelques années est le produit d'un système d'enseignement différent de la génération précédente. 
En effet, le Collège d'enseignement général et professionnel (C.E.G.E.P.) a remplacé l'ancien collège classique. Les étudiants issus du C.E.G.E.P. sont plus jeunes à leur arrivée à l'Université et possèdent une formation générale de base plus mince que les étudiants des années 60 . Par contre, ils sont plus ouverts à des expériences nouvelles, à une certaine imagination créatrice, et au sens de l'innovation.

Est-ce que l'approche pédagogique a été modifiée, compte tenu justement, du changement de clientèle?

La pédagogie des cours à notre École n'a pas été profondément modifiée ; toutefois, elle a dû s'adapter lentement au rythme d'apprentissage de la nouvelle génération d'étudiants. Ainsi, autrefois, loutil principal du professeur était le cours magistral et l'obligation pour les étudiants de lire abondamment dans le domaine criminologique. Dorénavant, les étudiants sont ouverts aux techniques audio-visuelles plutôt qu'à la lecture assidue et laborieuse. Nous tentons donc de nous réajuster un peu à ces nouveaux modes d'apprentissage, en recourant plus souvent aux dites techniques ainsi qu'à la formule du séminaire et des groupes de discussion. Toutefois, il est bien évident que la majorité des professeurs estiment qu'un certain nombre de *messages * criminologiques valables passent encore, et en très grand nombre, par le médium des journaux, des livres scientifiques et des revues savantes ; en conséquence, la télévision n'est pas prête à remplacer le livre, en criminologie tout du moins, nonobstant les oukases en ce sens du prophète de l'audio-visuel, Marshall McLuhan.

Croyez-vous qu'une association d'étudiants en criminologie est utile?

En principe, c'est comme la vertu, cela va de soi. Je pense qu'une telle association est absolument nécessaire afin de collaborer efficacement avec les professeurs sur les plans du renouveau pédagogique, du contenu des cours et du programme, des prises de position publiques sur des événements criminologiques importants... Malheureusement, comme nous le savons tous, ces associations étudiantes sont disparues, à toutes fins pratiques, de par la volonté des étudiants eux-mêmes, à la suite de la contestation générale estudiantine de 1968 au Québec. Si une telle association devait renaître en criminologie, je rappelle que l'Assemblée des professeurs de notre École a déjà accepté que le président élu d'un tel groupe puisse siéger avec nous. Je suis peut-être idéaliste, mais je pense qu'un étudiant, dont les opinions et les propositions seraient articulées, nuancées et modérées, tout en étant fermes quelquefois, jouirait d'un préjugé favorable au sein de l'Assemblée.

Lors d'une rencontre entre les étudiants et les professeurs de l'Ecole, en mars 1974, vous affirmiez que: * les professeurs ont besoin d'une certaine sécurité et d'une certaine stabilité de structure afin de préparer des cours adéquats ». Est-ce dire que certains cours sont remis en question par les étudiants ?

En fait, les étudiants remettent souvent en question moins les contenus de cours que la pédagogie. C'est leur droit le plus strict. Aussi, je crois que les réformes les plus importantes depuis deux ans à notre École l'ont été, précisément, sur le plan pédagogique. Dans cette perspective, les discussions avec les étudiants ont été très utiles à toute notre communauté criminologique. Lorsque je parle d'une structure stable, je parle du contenu des cours. En effet, chaque professeur remet à jour chaque année des parties plus ou moins importantes de son cours, selon 
l'état des travaux scientifiques de sa spécialité. S'il devait créer de novo, à neuf, ses enseignements presque à chaque année, afin de suivre la mode du moment, je pense qu'il deviendrait rapidement superficiel et la qualitê de son enseignement en serait affectée.

De plus, lors de cette même rencontre, vous affirmiez ce qui suit : a Je demande un respect ferme et intégral de notre pluralisme, en théorie et en pratique. * Pourtant deux mois plus tard, lors de la présentation du projet Bertrand-Tardif en vue de créer une nouvelle option dite sociopolitique, l'Assemblée des professeurs rejeta le projet. N'est-ce pas une négation?

Je ne le crois pas. En effet, le projet Bertrand-Tardif proposait deux changements importants : a) la mise sur pied d'une concentration dite socio-politique criminelle dès la première année du baccalauréat, et b) l'enseignement de deux grands cours-séminaires sur des matières qui n'avaient pas été jusqu'ici enseignées de façon explicite et adéquate à notre École, soit un cours de criminologie radicale ou critique et un cours de méthodologie qualitative. L'Assemblée a refusé, il est vrai, d'ouvrir une concentration socio-politique en première année, en croyant qu'un tel choix serait prématuré pour les nouveaux étudiants; en revanche, elle a reconnu que, de facto, il y avait deux orientations à l'école à partir de la seconde année du baccalauréat, soit une concentration en criminologie clinique et une autre en politique criminelle et elle a officiellement entériné les nouveaux plans de cours des professeurs Bertrand et Tardif qui ont d'ailleurs enseigné ces nouvelles matières dès le début de l'année académique 1974-1975.

Existe-t-il des relations entre l'Ecole de criminologie et les différents départements de l'Université de Montréal ?

La réponse est surtout négative. Évidemment, à l'occasion, et sur le plan purement administratif, nous participons à des mini-fronts communs; par exemple, en 1974-1975, nous avons mené une bataille commune avec l'Ecole de service social afin d'obtenir des budgets plus adéquats pour l'organisation des stages pratiques. De plus, il $y$ a un échango d'étudiants entre les différents départements de sciences humaines, en particulier, puisque les étudiants sont libres de choisir un certain nombre de cours à l'extérieur de leurs départements. Mais au niveau plus fondamental de la coopération active entre les professeurs des divers départements (prises de position publiques, séminaires ou recherches conjoints et intégrés), je dois avouer que les résultats sont maigres à ce chapitre.

Est-ce que les structures universitaires répondent, selon vous, aux besoins de l'Ecole ?

La structure la plus importante est celle de l'organisation des cours et des programmes puisque l'Université est d'abord caractérisée par un ensemble de programmes disciplinaires ou interdisciplinaires qu'elle affre " selon la "demande, et les besoins du milieu. La structure actuelle, à ce sujet, est satisfaisante. En effet, la Faculté des arts et des sciences (F.A.S.), par exemple, a une politique officielle souple, favorable à des programmes spécialisés, comme le nôtre, tout autant qu'à des programmes dits mineurs ou majeurs plus ouverts à un chassé-croisé entre les différentes disciplines. Le vrai problème, cependant, afin de favoriser cet échange entre les différentes disciplines, n'est pas un problème de structure, de programmes, mais plutôt d'agencement opérationnel pratique 
des horaires des cours afin d'éviter les conflits actuels d'horaires presque insurmontables si les différents départements n'y mettent pas un peu de bonne volonté.

Avez-vous l'impression que l'Ecole a contribué à apporter certains changements à l'Université ?

C'est une bien grande mission que vous semblez attribuer à l'f́cole de criminologie. Pour être franc, je ne vois pas d'impact spécial de notre École sur la structure générale de l'Université. Toutefois, je suis persuadé que la qualité de la présence des criminologues à l'Université de Montréal, que ce soit par le biais de l'enseignement, de la recherche ou des comités de l'Université où siègent des professeurs de notre École, a contribué à améliorer la qualité générale de la vie universitaire.

Avez-vous l'impression qu'à l'extérieur de l'Université, la criminologie a été un agent de changement social ?

Je suis profondément persuadé que la criminologie a été un agent réel de changement social au niveau de la justice en matière pénale et criminelle au Québec. Si la justice au Québec est aujourd'hui aussi civilisée et humaniste, sinon plus, que n'importe où en Amérique du Nord, je pense que le message criminologique véhiculé par les professeurs, les étudiants et les praticiens issus de notre Ecole a effectivement joué un rôle de premier plan. Par exemple : en 1960, les statistiques indiquaient que le Québec possédait le championnat peu glorieux des longues sentences à l'emprisonnement et était, de fait, la \& province * la plus punitive du Canada; en 1975, nous sommes maintenant la moins punitive. Sans l'impact de la criminologie, le Québec serait en retard, à mon avis, de vingt-cinq ans au chapitre de la philosophie pénale et de son action judiciaire.

Lorsque l'on analyse votre production académique, on est tenté de diviser cette dernière en deux périodes : une première période, de 1962 à 1970, où il est question surtout de déviance non institutionnalisée (déviance religieuse et criminalité des cols blancs), ainsi que d'une remise en question de la peine de mort. Une deuxième période, de 1970 a nos jours, où il est question cette fois-ci de déviance institutionnalisée (prisons, libérations conditionnelles, maison de transitions...). Acceptez-vous cette division (toujours arbitraire, il va de soi) ? Comment expliquez-vous ce passage? Votre engagement politique $y$ est-il pour quelque chose ?

Je n'ai pas assez de recul pour établir une telle dichotomie. Cette division, à mon avis, est de fait relativement arbitraire. En fait, l'évolution est plutôt dans le sens du passage d'un éclectisme de bon aloi dans les années 60 à des intérêts plus spécifiques avec les années 70 . Il est. normal qu'un jeune criminologue cherche sa voie pendant quelques années en regardant à droite et à gauche. Il est non seulement normal mais souhaitable, également, que le champ de vision criminologique puisse se rétrécir à un moment donné afin de livrer des réflexions plus en profondeur sur un certain nombre de problèmes. Je commence donc depuis quelques années à mieux délimiter ces intérêts; tout d'abord en optant pour des études et des recherches aptes à approfondir mes idées en politique criminelle et ainsi contribuer à une analyse critique du système de justice; ensuite, par un engagement politique dans un parti d'orientation sociale-démocrate qui me permet d'expliciter mes conclusions dans un programme structuré de réformes politiques de l'administration de la justice. 
Vous affirmiez, en 1972, que : Le Solliciteur général du Canada est, ni plus ni moins, que le ministère de la criminologie s, donnerez-vous ainsi raison à ceux qui maintiennent que la criminologie québécoise est la - servante des appareils répressifs de l'Etat s?

Cette expression prête, en effet, à confusion. En fait, je voulais tout simplement indiquer qu'au niveau gouvernemental, le ministère du Solliciteur général du Canada est l'unité administrative dont les intérêts généraux recoupent plusieurs des intérêts des professeurs et des étudiants de notre École puisque ce ministère est responsable des pénitenciers, des libérations conditionnelles, de la Gendarmerie royale du Canada, de la loi des jeunes délinquants... De plus, il est le seul organisme gouvernemental qui possède un service de recherche et de documentation criminologique vraiment structuré. Je voulais donc simplement signaler que des échanges entre l'École et les fonctionnaires du Solliciteur général étaient valables dans la mesure où certains domaines criminologiques nous intéressaient mutuellement. Cependant, il n'est aucunement question de sacrifier notre liberté critique vis-à-vis le gouvernement sur le contenu même des politiques criminelles qu'il patronne. Pour ma part, $j$ 'ai critiqué très sévèrement ces dernières années les politiques gouvernementales autant du ministère du Solliciteur général du Canada que celles du ministère de la Justice du Québec. Par conséquent, la criminologie de notre École se veut utile a la société sans être la servante de qui que ce soit.

Vous avez proposé en 1968, l'application an Québec d'un nouvel indice de la criminalité, celui de Sellin et Wolfgang. Cet indice ne risque-t-il pas de rendre la répression plus sévère et plus subtile?

Il faut nous rappeler que le thème des statistiques criminelles était un thème criminologique important dans les années 60 , non seulement au Québec mais également en Amérique du Nord et en Europe. J'ai donc travaillé le sujet à cette époque parce qu'il était jugé important par la communauté scientifique et parce que le hasard m'a placé alors au contact direct des deux criminologues américains, Sellin et Wolfgang, qui ont développé les idées les plus originales sur ce thème au cours des vingt-cinq dernières années. Mon objectif était d'abord d'ordre théorique et méthodologique : mieux connaître la réalité criminelle afin de mieux comprendre le phénomène criminel: d'ordre pratique, également, puisqu'une meilleure connaissance de la distribution, en quantité et en qualité, de la criminalité peut évidemment servir les agents policiers afin de mieux répartir leurs énergies et d'obtenir des résultats plus concluants. Imaginer que cet indice contribue à rendre la répression plus sévère et plus subtile me semble une réaction inappropriée.

Quelles relations existent entre le monde de la police et l'Ecole de criminologie?

Je veux être très explicite sur ce sujet. Il n'y a aucun lien direct, manifeste ou caché, entre les forces de l'ordre et l'École. Nous ne formons, ni de près ni de loin, de futurs policiers. Toutefois, quelques cours et séminaires abordent le monde de la police dans le sens d'une réflexion sociologique sur le travail des policiers et sur l'administration de la police. Cette réfiexion est absolument indispensable à une compréhension globale de l'administration de la justice en matière pénale et criminelle puisque l'organisation policière est un a morceau " important du système et que des propositions de réformes du système de justice doivent englober le sous-système policier, ipso facto. Le monde de la police est un élément crucial de toute politique criminelle articulée. 
Quelles différences établissez-vous entre la criminologie, la psychologie, la psycho-éducation, au niveau universitaire, et les techniques auxiliaires de la justice ou les techniques d'éducation spécialisée au niveau du C.E.G.E.P.?

Je crois que la concentration en criminologie clinique de notre Ecole est en fait une synthèse originale, même si elle est encore à sa période d'adolescence disciplinaire, d'une psychologie appliquée ainsi que du service social spécialisé en matière de délinquance et de criminalité. Cette synthèse que nous bâtissons est le véritable défí des cliniciens et des praticiens de la criminologie. En ce qui concerne, par ailleurs, la concentration en politique criminelle, une synthèse différente est bâtie depuis quelques années, à partir des perspectives de base de la sociologie, du droit et des sciences politiques. Il n'y a plus, de toute façon, à mon avis, de discipline à l'état pur. Il y a une véritable dialectique d'approches divergentes et convergentes qui est absolument nécessaire pour comprendre dans sa globalité les phénomènes de déviance et de criminalité. La criminologie, sans renier ses allégeances naturelles avec les autres disciplines de sciences humaines et sociales, doit, toutefois, poursuivre un chemin et un développement personnel que nulle autre discipline de base ne peut accomplir à sa place puisque la criminologie est la seule approche disciplinaire totale et intégrative du phénomène criminel. La psychiatrie, la psychologie, la psycho-éducation et le service social possèdent, tous et chacun, des perspectives intéressantes sur le phénomène criminel et ils ont une contribution à nous apporter. Mais la synthèse théorique et méthodologique de la criminologie est particulière et essentielle. Le criminologue diplômé en criminologie au niveau universitaire est donc mieux préparé, règle générale, à travailler dans le secteur de la justice en matière pénale et criminelle, aux plans clinique et politique, que tout autre praticien des sciences de l'homme. J'avoue, évidemment, que ce jugement est relatif et historique, Il s'applique à la situation actuelle telle que je la perçois. In n'est pas dit qu'une telle synthèse originale ne puisse se réaliser ailleurs dans le monde ou dans un avenir plus ou moins rapproché dans un cadre et un contexte différents. Ce jugement est valable, également, pour le niveau collégial, où les < techniques de ... > constituent en fait une synthèse primaire pré-universitaire dont la valeur ne peut encore être jugée puisque ces programmes collégiaux n'existent que depuis quelques années.

Comme vous le savez, plusieurs criminologues ont tendance à percevoir dans votre manifeste sur s la justice criminelle au Québec : 1975-1980, une orientation future de la criminologie; en particulier lorsque vous affirmez que les * secteurs clés de contrôle de la criminalité doivent être les suivants, par ordre prioritaire : a. Les infractions contre la qualité de la vie (criminalité de la route et du travail, protection du consommateur, pollution...) ; b. La criminalité en col blanc (fraudes et corruptions économiques et politiques...); c. Le crime organisé, le crime professionnel; d. La criminalité violente et impulsive contre les personnes et les biens." L'orientation idéologique du programme de l'Ecole de criminologie permetelle, toutefois, une telle perspective ?

Je pense que oui, définitivement. Évidemment, je suis conscient que les professeurs et les praticiens de criminologie clinique continueront d'axer leurs efforts surtout au palier de la criminalité violente et impulsive contre les personnes et les biens. C'est leur droit le plus strict. En retour, les professeurs et les chercheurs en politique criminelle de l'École ont déjà amorcé depuis un an ou deux un virage à gauche important qui les amène de plus en plus à poursuivre des intérêts théoriques et des recherches empiriques aux paliers des délits contre la qualité de la vie ainsi que 
de la criminalité économique. Ce n'est qu'un début modeste, je l'avoue; cependant, je pense que le virage est nettement pris et qu'il n'y aura pas de retour en arrière.

Une dernière question, si vous me le permettez. Comment percevez-vous l'Association professionnelle des criminologues du Québec (A.P.C.Q.)?

L'A.P.C.Q. est jeune. Elle fut fondée en 1968. Au début, il s'agissait à toutes fins pratiques d'un autre groupement universitaire et academique puisque les premiers présidents, et j'en suis (1969-1971), furent des professeurs de notre École. Toutefois, avec l'arrivée massive des praticiens, en 1971, une orientation plus marquée s'est fait jour, et c'est normal, pour organiser l'A.P.C.Q. autour d'objectifs d'action plus pratiques, plus près des préoccupations quotidiennes des praticiens et des cliniciens de la * criminologie sur le terrain *. Quelques sessions de recyclage et quelques prises de position publiques ont été réalisées à l'initiative de I'A.P.C.Q. depuis quelque temps. Cependant, le bilan global des activités de l'A.P.C.Q. est relativement maigre. Cela s'explique. En effet, sans véritables ressources financières et sans personnel permanent, ni professionnel, ni de secrétariat, l'A.P.C.Q. n'a pu exercer un rôle réel de leadership dans le champ de la criminologie appliquée. Je pense qu'il est temps que l'A.P.C.Q. se dote d'une organisation sérieuse et permanente. Après tout, l'école de criminologie a maintenant à son actif plus de 500 diplômés en criminologie depuis 1960 , date de sa fondation.

Après plus de 15 ans de développement actif au Québec, la criminologie québécoise ne peut plus prétendre encore qu'elle est trop jeune pour entreprendre des travaux d'envergure. Nous avons maintenant les structures, les programmes et les hommes nécessaires que nous voulions. La crédibilité de la criminologie québécoise se jouera véritablement au cours des prochaines années. À nous de relever le défi !

\section{BIBLIOGRAPHIE}

A. Références générales citées dans le texte.

MERTON, R. (1957), Social Theory and Social Structure, New York, Free Press.

MILLS, C.W. (1959), The Sociological Imagination, New York, Oxford University Press.

PARSONS, T. (1951), The Social System, New York, Free Press.

ROBERT, P. (1973), - La sociologie entre une criminologie de passage à

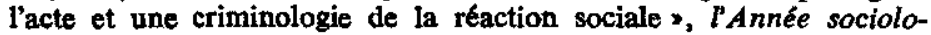
gique, 24, 441-504.

SELLIN, T., et WOLFGANG, M.E. (1964), The Measurement of Delinquency, New York, Wiley.

VOLD, G. (1958), Theoretical Criminology, New York, Oxford University Press.

B. Références aux travaux de l'auteur citées dans le texte.

NORMANDEAU, A. (1964), * Une théorie économique de la révolution au Québec *, Cité libre, avril, 9-14.

NORMANDEAU, A. et DÉSY, J. (1964), La secte de Saint-Jovite : un phénomène de pluralisme vertical $\gg$, Cité libre, mai, 22-27. 
NORMANDEAU, A. (1965), - Les deviations en affaires et le crime en col blanc , Revue internationale de criminologie et de police technique, octobre, 247-258.

NORMANDEAU, A. (1966), - La peine de mort au Canada ", Revue de droit pénal et de criminologie, mars, 547-559.

NORMANDEAU, A (1967), - La criminologie : pot-pourri ou discipline scientifique distincte?, Revue canadienne de criminologie, juillet, 257-264.

NORMANDEAU, A. et AKMAN, D. (1968), * Towards the measurement of criminality in Canada s, Acta Criminologica, janvier, 135-260.

NORMANDEAU, A. (1969), * L'homme psychologique et la culture du Xxe siècle s, Maintenant, février, 48-52.

NORMANDEAU, A. (1969), \&ioneers in criminology >, Journal of criminal law, criminology and police science, march, 28-32.

NORMANDEAU, A. et FATTAH, E. (1969), Sondage d'opinion publique sur la justice criminelle au Quebec, Québec, Editeur officiel.

NORMANDEAU, A. (1970), * Politique et réforme pénitentiaire *, Revue de science criminelle et de droit pénal comparé, juillet, 605-621.

NORMANDEAU, A. (1971), - Socialisation de la justice au Québec ", Relations, avril, 103-105.

NORMANDEAU, A., LE BLANC, M. et SZABO, D. (1971), Applied criminology and government policy ,, Issues in criminology, winter, 55-83.

NORMANDEAU, A. (1972), Violence and Robbery *, Acta Criminologica, janvier, 11-106.

NORMANDEAU, A. (1972), \& La criminologie québécoise, parlons-en ! ", Maintenant, août, 12-14.

NORMANDEAU, A. et LANDREVILLE, P. (1973), se criminel et la société canadienne, version Allmand ", le Devoir, 22 décembre, 5.

NORMANDEAU, A. et al. (1973), e Protestation de groupes, violence et système de justice criminelle ", in $\mathrm{D}$. Szabo, la Criminalité urbaine (pp. 47-81), Montréal, P.U.M.

NORMANDEAU, A. et al. (1974), r Police et violence collectives, in D. Szabo, Police, culture et société (pp. 91-158), Montréal, P.U.M.

NORMANDEAU, A. (1975), \&a justice criminelle au Québec : 1975-80 *, Criminologie made in Canada, janvier, 75-86.

NORMANDEAU, A., RICO, J. et TARDIF, G. (1975), Le livre blanc de Choquette vu par trois criminologues „, le Devoir, 24 mai, 5-6.

NORMANDEAU, A. (1975), * Pour une justice pénale mieux axée sur les formes significatives de criminalité 3, le Devoir, 15 septembre, 5 .

NORMANDEAU, A. (1976), - Pour une justice sociale-démocrate au Canada, au Québec et ailleurs *, Criminologie made in Canada, janvier, 137-151. 